\section{Refugee Documentation Centres Meet}

(Geneva, June 27 - July 1, 1983)

For the first time in history, a meeting of centres devoted to the collection and dissemination of refugee research and information was held in Geneva under the auspices of the IRIRC (International Refugee Integration Resources Centre) at the Institut Henri-Dunant in Geneva. Representatives of 13 centres (see box) from nine different countries as well as a number of observers from international agencies met to discuss modes of cooperation.

Some centres, such as the Swedish Immigration Board, are government centres (GOs). Others, such as the Australian, were non-government organizations (NGOs). Still others, such as the German, were quasi governmental organizations (Quango's). They also varied in the range of coverage. Some took on the full mandate of migration studies, others, the smaller mandate of displaced persons, and still others restricted themselves to refugees.
Some of these were even more specialized, concentrating on Southeast Asian refugees or only refugees seeking refugee status in the courts in contrast to those involved in mass movements.

In addition to becoming acquainted with each other's activities, the documentation centres agreed to set up an informal network in which the refugee documentation centre in each country would supply all other documentation centres with information and documents produced in its country. These would include: copies of very significant documents available free; publications of the member; lists of important publications available for purchase; abstracts of key documents of that country; an index of all other material produced in that country and acquired or located by the member with a key word index for easy reference; a list of refugee organizations and refugee-assisting organizations in that country.

IRIRC as an international clearing house, will be responsible for providing a working thesaurus for indexing, and copies of very significant international documents. In addition, it would publish abstracts and a comprehensive index keyworded for easy reference as well as filling in gaps where a country lacked a full capacity documentation centre.

The symposium also passed a resolution urging that "in the planning and budgeting of specific programs, assessment of information, documentation, and research requirements should be made and appropriately supported."

The Refugee Documentation Project invites submissions of

- Abstracts

- Documents

- Reports

- Publications

- Articles

- Names of organizations

- Names of experts.

The RDP will arrange for their redistribution internationally.

\begin{tabular}{|c|c|c|c|c|c|}
\hline Full Name & Acronym & Coverage & Library & Publications & $\begin{array}{l}\text { Technical } \\
\text { References }\end{array}$ \\
\hline $\begin{array}{l}\text { Clearing House on Migration } \\
\text { issues }\end{array}$ & CHOMI (Australia) & migration & 32 hours weekly & $\begin{array}{l}\text { - quarterly abstracts } \\
\text { - bibliographies } \\
\text { - a magazine Migration Action } \\
\text { - occasional papers and reprints }\end{array}$ & none \\
\hline Refugee Documentation Project & RDP (Canada) & refugees & 35 hours weekly & $\begin{array}{l}\text { - bibliographies } \\
\text { - periodical, Refuge } \\
\text { - occasional papers } \\
\text { - research reports }\end{array}$ & $\begin{array}{l}\text { microcomputer } \\
\text { d-Base II }\end{array}$ \\
\hline \multirow[t]{2}{*}{ Danish Refugee Council } & DRC (Denmark) & not known & not known & - not known & not known \\
\hline & CeDrasemi (France) & $\begin{array}{l}\text { Southeast Asian } \\
\text { refugees }\end{array}$ & Research only & $\begin{array}{l}\text { - bibliographies } \\
\text { - monographs (12) }\end{array}$ & no \\
\hline $\begin{array}{l}\text { Zentrale Documentations stelle Der } \\
\text { Freien Wohlfahrtspflege Fur } \\
\text { Fluchtling, e.v. }\end{array}$ & ZDWF (Germany) & $\begin{array}{l}\text { specializes in } \\
\text { refugeesandasylum } \\
\text { seekers in West } \\
\text { Germany }\end{array}$ & specialized library & $\begin{array}{l}\text { - relevant jurisprudence } \\
\text { - countries of origin } \\
\text { - keyword catalogue }\end{array}$ & $\begin{array}{l}\text { microfilm EDP data } \\
\text { bank }\end{array}$ \\
\hline Displaced Persons Centre & DPC (Netherlands) & $\begin{array}{l}\text { refugees in } \\
\text { Southeast Asia }\end{array}$ & & $\begin{array}{l}\text { - periodic Review } \\
\text { - special Reports } \\
\text { - monographs }\end{array}$ & no \\
\hline $\begin{array}{l}\text { Human Rights Information and } \\
\text { Documentation Systems }\end{array}$ & $\begin{array}{l}\text { HURIDOCS } \\
\text { (Netherlands) }\end{array}$ & human rights & a co-op network & - Thesaurus on human rights & yes \\
\hline $\begin{array}{l}\text { Statens Invandrarverk (Swedish } \\
\text { Immigration Board) }\end{array}$ & SIB in general & migration & yes & - not known & not yet \\
\hline \multirow[t]{2}{*}{ Cooperative Européenne Longo Mai } & $\begin{array}{c}\text { CEDRI } \\
\text { (Switzerland) }\end{array}$ & $\begin{array}{l}\text { Turkish \& Kurdish } \\
\text { refugees and } \\
\text { immigrants in } \\
\text { Europe }\end{array}$ & no & - Information Bulletin & no \\
\hline & $\begin{array}{c}\text { CREDIS } \\
\text { (Switzerland) }\end{array}$ & $\begin{array}{l}\text { research and } \\
\text { documentation on } \\
\text { refugee resettlement } \\
\text { in Sweden }\end{array}$ & yes & - monographs & no \\
\hline $\begin{array}{l}\text { International Refugee Integration } \\
\text { Resource Centre }\end{array}$ & IRIRC (Switzerland) & $\begin{array}{l}\text { refugees with a } \\
\text { focus on settlement } \\
\text { issues }\end{array}$ & yes & $\begin{array}{l}\text { - Refugee Abstracts } \\
\text { - bibliographical indexes }\end{array}$ & data bank \\
\hline $\begin{array}{l}\text { Association for the Study of the } \\
\text { World Refugee Problem }\end{array}$ & $\begin{array}{c}\text { ASWRP } \\
\text { (Switzerland) }\end{array}$ & & & & \\
\hline Center for Migration Studies & CMS (U.S.) & migration & yes & $\begin{array}{l}\text { - International Migration Review, } \\
\text { special issues, monographs }\end{array}$ & not yet \\
\hline
\end{tabular}

